\title{
BMJ Open Outcome Prognostication of Acute Brain Injury using the Neurological Pupil Index (ORANGE) study: protocol for a prospective, observational, multicentre, international cohort study
}

\author{
Mauro Oddo, ${ }^{1,2}$ Fabio Taccone, ${ }^{3}$ Stefania Galimberti, ${ }^{4,5}$ Paola Rebora, ${ }^{4,6}$ \\ Giuseppe Citerio (D) , 4,7 on behalf of the Orange Study Group
}

To cite: Oddo $\mathrm{M}$, Taccone $\mathrm{F}$, Galimberti S, et al. Outcome Prognostication of Acute Brain Injury using the Neurological Pupil Index (ORANGE) study: protocol for a prospective, observational multicentre, international cohort study. BMJ Open 2021;11:e046948. doi:10.1136/ bmjopen-2020-046948

- Prepublication history and supplemental material for this paper is available online. To view these files, please visit the journal online (http://dx.doi org/10.1136/bmjopen-2020046948).

Received 14 November 2020 Revised 03 March 2021 Accepted 27 April 2021

Check for updates

(C) Author(s) (or their employer(s)) 2021. Re-use permitted under CC BY-NC. No commercial re-use. See rights and permissions. Published by BMJ.

For numbered affiliations see end of article.

Correspondence to Professor Giuseppe Citerio; giuseppe.citerio@unimib.it

\section{ABSTRACT}

Introduction The pupillary examination is an important part of the neurological assessment, especially in the setting of acutely brain-injured patients, and pupillary abnormalities are associated with poor outcomes. Currently, the pupillary examination is based on a visual, subjective and frequently inaccurate estimation. The use of automated infrared pupillometry to measure the pupillary light reflex can precisely quantify subtle changes in pupillary functions. The study aimed to evaluate the association between abnormal pupillary function, assessed by the Neurological Pupil Index (NPi), and long-term outcomes in patients with acute brain injury (ABI). Methods and analysis The Outcome Prognostication of Acute Brain Injury using the Neurological Pupil Index study is a prospective, observational study including adult patients with $A B I$ requiring admission at the intensive care unit. We aimed to recruit at least 420 patients including those suffering from traumatic brain injury or haemorrhagic strokes, over 12 months. The primary aim was to assess the relationship between NPi and 6-month mortality or poor neurological outcome, measured by the Extended Glasgow Outcome Score (GOS-E, poor outcome=GOS-E 1-4). Supervised and unsupervised methods and latent class mixed models will be used to identify patterns of NPi trajectories and Cox and logistic model to evaluate their association with outcome.

Ethics and dissemination The study has been approved by the institutional review board (Comitato Etico Brianza) on 16 July 2020. Approved protocol V.4.0 dated 10 March 2020. The results of this study will be published in peerreviewed journals and presented at conferences. Trial registration number NCT04490005.

\section{INTRODUCTION}

Pupillary examination, including pupillary light reactivity (PLR), is a fundamental part of the clinical examination in patients suffering from acute brain injury (ABI), with both diagnostic and prognostic values. ${ }^{1}$ As an example, the oculomotor nerves might be compressed due to displacement of the brainstem, and

\section{Strength and limitation of this study}

- The study will cover the more common neurological emergencies and, in a large population of patients with acute brain injury, the relationship between pathological Neurological Pupil Index and outcome.

- The standard data acquisition in the centres, transferred from the device into the eCRF, and the granularity of data will guarantee high-quality data.

- Due to the observational nature of our study, we will report only associations and not causality relationship.

clinicians have accepted fixed and dilated pupils as part of the 'herniation' syndrome.

Elevated intracranial pressure (ICP) may alter midbrain function and cause abnormalities in pupil size, symmetry and PLR. ${ }^{2-5}$ Sustained or newfound pupillary abnormalities are associated with a worse outcome, ${ }^{6}$ and indeed PLR is a robust validated predictor in several prognostic models, such as the Corticosteroid Randomization after Significant Head Injury and the International Mission for Prognosis and Analysis of Clinical Trials (IMPACT) scores. ${ }^{7}$ However, in current clinical practice, the pupillary examination is performed using a manual, hand-held light source (eg, pen torch), implying that the evaluation of pupillary size and reactivity remains essentially based on a visual qualitative assessment. This traditional approach has several limitations, such as limited precision (eg, due to small pupil size or specific administered treatments), significant intraobserver and interobserver variability, differences in ambient light exposure between measurements or the technique used to direct the stimulus (ie, intensity, proximity, duration and orientation of the light source)..$^{8-10}$ 
Quantitative, automated, infrared technology for pupillary examination has been used for years in ophthalmology and anaesthesiology research. ${ }^{411}{ }^{12}$ Its interest in neurocritical care has progressively grown, ${ }^{13}$ in parallel with the advancements in device technology. In this regard, the use of the non-invasive NPi-200 pupillometer (Neuroptics, Laguna Hills, California, USA) allows the measurement of a series of dynamic pupillary variables which can be integrated into an algorithm to compute the Neurological Pupil Index (NPi). The NPi is calculated by the handheld device using a set of variables including size, latency, constriction velocity and dilation velocity. Each variable from an individual pupil measurement is compared against the mean of a reference distribution of healthy subjects, taking the difference and then standardising it by the corresponding SD. Finally, the sets of all the standardised differences (or z-scores) are combined to fall into a scale set between 0 and 5 (with a 0.1 decimal precision), ${ }^{2}$ with an NPi value of $<3$ indicating abnormal pupillary reactivity. ${ }^{2314} \mathrm{NPi}$ is not influenced by sedationanalgesia, at the doses used in neurocritical care practice, and by mild hypothermia. ${ }^{2}{ }^{14}$ Preliminary single-centre data recently demonstrated that abnormal NPi is associated with worse outcome in patients with traumatic ${ }^{15}$ and haemorrhagic ABI, ${ }^{16}{ }^{17}$ and can be a useful adjunct for ICP monitoring ${ }^{15}$ and response to therapy. ${ }^{18}$ There is currently a great need for quantitative tools to predict early prognostication in patients with $\mathrm{ABI}$, and the NPi appears of potentially great value. ${ }^{19}$

For this purpose, large multicentre studies are required. We recently conducted an international multicentre study that demonstrated the prognostic value of $\mathrm{NPi}$ in the setting of early prognostication of ABI following cardiac arrest. ${ }^{20}$ Given the encouraging results, we decided to enlarge the spectrum of ABI diseases monitored with NPi in a new study. Here, we aim at evaluating the prognostic value of the NPi in patients with $\mathrm{ABI}$ following traumatic brain injury (TBI), aneurysmal subarachnoid haemorrhage $(\mathrm{SAH})$ or intracerebral haemorrhage (ICH) at risk of secondary ICP elevation.

\section{Objectives}

The primary aim of the study was to evaluate the association between abnormal NPi and long-term outcomes (ie, 6-month mortality and neurological outcome, measured using the Extended Glasgow Outcome Score (GOS-E)) in patients with $\mathrm{ABI}$.

The secondary aim, in patients with ICP monitoring, was to evaluate the relationship of abnormal NPi and ICP following ABI.

\section{METHODS AND ANALYSIS}

\section{Study design and setting}

This is a prospective, observational cohort study involving 13 centres worldwide that routinely use pupillometry. Recruitment will last 12 months, and patients will be followed up for 6 months.

\section{Study population}

Consecutive participants will be recruited at the participating centres according to the inclusion and exclusion criteria reported as follows.

\section{Inclusion criteria}

- Intensive care unit (ICU) admission after ABI, including TBI, SAH and ICH requiring intubation and ventilation for neurological reasons/deterioration.

- Age $\geq 18$ years old.

- Pupillometry is available as a standard evaluation tool.

\section{Exclusion criteria}

- Facial trauma not allowing pupils' evaluation.

\section{Screening and data collection}

All patients admitted to the participating ICUs after ABI will be screened daily and entered into a screening $\log$ (online supplemental appendix, Screening LogRegistry). Each ICU will recruit consecutive eligible patients and collect data for each included patient daily in an expanded electronic CRF developed in REDCap (online supplemental appendix, eCRF).

Both common data elements and aetiology-specific data will be recorded. Demographic characteristics and medical history information will be extracted from patients' medical records including gender, age, comorbidities, diagnosis, timeline and clinical presentation of ABI. All NPi and ICP data, as well as additional neuromonitoring and neuroimaging data, will be extracted from patients' medical records too and documented in the eCRF. The patients admitted to the units will be screened by research staff, and the pupillometry evaluation, part of the clinical practice in all the centres, will be performed by trained staff. The two eyes' specific NPi and the matched ICP will be collected every 4 hours from admission up to day 7. Data collected will also include additional ICPderived variables (eg, ICP max, ICP 20 index: number of end hourly measures of ICP of $>20 \mathrm{~mm} \mathrm{Hg}$ divided by the total number of measurements, multiplied by 100) and interventions (eg, osmotherapy, therapy intensity level and neuroimaging). The GOS- $\mathrm{E}^{21}$ will be collected at ICU/hospital discharge and 6 months from ICU admission. The latter will be collected via telephone-structured interviews with patients and/or family members using a validated questionnaire. ${ }^{21}$ In case of death, details on the cause and date will be collected as well.

\section{Sample size and statistical analysis}

As no data on NPi trajectory in time and its association with outcome is available for sample size calculation, we referred to pupil reactivity as a proxy of NPi behaviour and to results of a study recruiting patients with similar characteristics (Intracranial Pressure monitoring in the ICU: An International Prospective Observational Study on Intracranial Pressure in Intensive Care, trial registration number: NCT03257904). Assuming a 6-month mortality of $53 \%$ in patients with one or both unreactive pupils and $29 \%$ in patients with both reactive pupils, a sample of 420 
patients achieves $94 \%$ power to detect a HR of nearly 2 at a 0.05 significance level. As far as recruitment, each of the 13 centres involved in the study is expected to include nearly 35 patients in a recruitment period of 1 year, and this is in line with their potentiality of recruitment for the three pathologies that is of at least 80 patients per year.

Qualitative variables will be summarised by counts and percentages, while quantitative characteristics will be summarised by quartiles or mean and SD, as appropriate. Supervised and unsupervised methods (eg, pattern recognition and cluster trajectory analyses) will explore the possibility to identify patterns of NPi trajectories associated with different prognosis on the individual NPi longitudinal measurements. NPi trends will be also modelled by longitudinal mixed models using splines and latent class mixed models.

A Cox and a logistic model will be applied to evaluate the association between the NPi process and the 6 month mortality and neurological recovery (GOS$\mathrm{E} \leq 4$ vs GOS-E $>4$ ) at 6 months, respectively. This will be done considering NPi in categories that identify different potential patterns in NPi longitudinal profiles or using summary measures that have been already introduced in this context, such as the percentage of $\mathrm{NPi}<3$ observed in the time course or the area under the trajectory in time. This analysis will be done both overall, on a multivariable model that will explore the interaction with the different pathologies, and on the three specific pathologies to account for the disease-specific risk factors (eg, the components of the IMPACT model for patietns with TBI). The two eyes will contribute to these analyses with the worst result only and with their absolute NPi difference, but multivariate models will be also investigated to consider data from both eyes as a sensitivity analysis. To evaluate the association between NPi and ICP, a multivariable longitudinal linear model on the ICP20 index (percentage of end-hourly measures of ICP $>20 \mathrm{~mm} \mathrm{Hg}$ ) will be applied on patients with ICP monitoring, both overall and on the three diseases. A longitudinal linear regression model on ICP values will be also considered. All the analyses will be performed in R.

\section{Patient and public involvement}

It was not appropriate or possible to involve patients or the public in the design, conduct, reporting or dissemination plans of our research.

\section{Limitations}

The main limitation of this study is its observational nature, which makes it impossible to draw causal inferences reliably. We try to overcome this limitation with a preplanned statistical plan and a rigorous analysis of the findings.

\section{ETHICS AND DISSEMINATION}

This study will be conducted in compliance with the protocol V.4.0 dated 10 March 2020 approved by the ethics committee 'Brianza' at the ASST-Monza (approval date: 16 July 2020). Each National Coordinator will notify the relevant ethics committee, in compliance with the local legislation and rules. The approval of the protocol (if required by local authorities) must be obtained before any participant is enrolled. Any amendment to the protocol will require review and approval by the three chief investigators before the changes are implemented into the study. In case of patients not able to provide an informed consent at the time of study recruitment, each country will refer to the local/national law on the matter of lack of capacity. Generally, if patients will regain capacity at the follow-up, they will be asked to provide the informed consent for the acute data and follow-up or deny further research participation without any objection against use for research of data collected during the acute phase or deny further research participation and require the destruction of acute data collected. The study will be performed according to the Helsinki Declaration and the International Conference on Harmonisation for Good Clinical Practice.

The study will be published in a peer-reviewed journal and presented at the main intensive care medicine scientific congresses. Authorship will be granted according to the International Committee of Medical Journal Editors (ICMJE) definitions.

The University of Milano-Bicocca has the property of all the data collected. The data reside at the University Milano-Bicocca as study sponsor; all procedures will comply with the EU regulation on data protection 2016/679 on the protection of natural persons regarding personal data processing and movement. Local site data will be co-owned by each participating centre, and they will be given access to local data for any scientific purpose on request. By entering data into the Outcome Prognostication of Acute Brain Injury using the Neurological Pupil Index (ORANGE) study database, each centre agrees that the chief can use these data for scientific purposes. Any requests for the use of the data set for subsequent studies will be made to the ORANGE study chief investigators. Any requests for the use of the data set for subsequent studies will be made to the ORANGE study chief investigators. A formal data monitoring committee is not needed since it is not an interventional controlled study. A dedicated staff from the University of Milano-Bicocca will monitor the data included in the eCRFs checking for inconsistencies.

\section{Data confidentiality}

Participant confidentiality is strictly held in trust by the participating investigators and their staff. All medical or administrative staff with access to the data is subject to a duty of confidentiality and data protection. Therefore, the study protocol, documentation, data and all other information generated will be held in strict confidentiality agreement protocols. The study sponsor and representatives of local authorities may inspect all documents and records required to be maintained by the local 
investigator for the participants in this study. The clinical study site will permit access to such records.

Study participant research data, which is for purposes of statistical analysis and scientific reporting, will be transmitted to the Data Manager and the Statisticians of the study. For this purpose, data will be deidentified at input into the eCRF by the local centres/investigators. Individual participants and their research data will be identified by a unique study identification number. The eCRF system used by clinical sites and by research staff will be secured.

\section{Lack of capacity and delayed consent}

Patients recruited in this study will not be able to provide informed consent at the time of recruitment. ${ }^{22}$ Consent procedures will follow local policies.

At follow-up, patients who have regained capacity will be asked to provide informed consent and will be given the possibility to

- Provide informed consent for the acute data and follow-up.

- Deny research participation and request destruction of acute data collected.

\section{Medical care related to the study}

The medical care of the participant in the study is performed as per the local standard of care, without any deviation from clinical protocols. All the procedures follow the latest recommendations for ABI.

\section{Premature termination or suspension of study}

This study may be suspended or prematurely terminated for reasonable cause agreed by the investigators. Written notification, documenting the reason for study suspension or termination will be provided by the suspending or terminating party. If the study is prematurely terminated or suspended, the local principal investigator will promptly inform the ethics committees or other local authorities according to local legislation and will provide the reasons for the termination or suspension. Circumstances that may warrant termination could be recruitment that will be prolonged for $>5$ years or insufficient compliance with the protocol. The study may resume when the concerns have been addressed and issues resolved.

\section{Author affiliations}

'Department of Intensive Care Medicine, CHUV, Lausanne, Switzerland

${ }^{2}$ Medical Direction, CHUV, Lausanne, Switzerland

${ }^{3}$ Department of Intensive Care, Université Libre de Bruxelles, Bruxelles, Belgium

${ }^{4}$ School of Medicine and Surgery, University of Milano - Bicocca, Monza, Italy

${ }^{5}$ Bicocca Bioinformatics Biostatistics and Bioimaging B4 Center, Università degli Studi di Milano-Bicocca, Monza, Italy

${ }^{6}$ Bicocca Bioinformatics Biostatistics and Bioimaging B4 Center, University of Milan-

Bicocca Faculty of Medicine and Surgery, Monza, Italy

${ }^{7}$ Neurolntensive Care Unit, ASST di Monza, Monza, Italy

\section{Twitter Giuseppe Citerio @dr_Cit}

Collaborators The Orange study group: Badenes R, University of Valencia Hospital Clinic, Anesthesiology and Surgical-Trauma Intensive Care, Valencia, Spain; Bouzat P, Department of Anesthesia and Intensive Care, Grenoble-Alpes University Hospital (CHU), Grenoble, France; Caricato A, Department of Anesthesiology and
Intensive Care Medicine, Fondazione Policlinico Universitario Agostino Gemelli IRCCS, Università Cattolica del Sacro Cuore, Rome, Italy; Chesnut RM, Department of Neurological Surgery, Harborview Medical Center, University of Washington, Seattle, USA and Department of Orthopaedic Surgery, Harborview Medical Center, University of Washington, Seattle, USA; Hemphill C, Department of Neurology, University of California San Francisco, San Francisco, CA, USA; Rasulo F, Intensive Care and Anesthesiology, Department of Surgical Specialties, Radiological Sciences and Public Health, University of Brescia and Spedali Civili Hospital, Brescia, Italy; Schwab S, Department of Neurology of the Friedrich-Alexander-University ErlangenNuremberg, Germany; Suarez JI, Division of Neurosciences Critical Care, The Johns Hopkins University School of Medicine, Baltimore, Maryland; Sunde K, Department of Anaesthesiology, Oslo University Hospital, Ullevål, Oslo, Norway and Institute of Clinical Medicine, University of Oslo, Institute of Clinical Medicine, Oslo, Norway; Aaron Blandino, Department of Intensive Care Medicine, Ramón Y Cajal University Hospital, Madrid, Spain; Letterio Malgeri, School of Medicine, University of Messina, Messina, Italy; Eleonora Rossi, School of Medicine, University of Pavia, Pavia, Italy; Alessia Vargiolu, School of Medicine, University of Milano-Bicocca, Monza, Italy; Francesca Elli, School of Medicine, University of Milano-Bicocca, Monza, Italy; Francesca Graziano, School of Medicine and Surgery, University of Milano-Bicocca, Milan, Italy and Bicocca Bioinformatics Biostatistics and Bioimaging B4 Center, University of Milano-Bicocca, Monza, Italy.

Contributors M0, FT and GC conceived and designed the study. SG and PR conceived the statistical plan of the study. SG and GC drafted the manuscript. All authors revised the manuscript and gave the final approval of the version to be submitted.

Funding University Milano-Bicocca is the study's sponsor. The study was supported by an unrestricted grant from Neuroptics.

Competing interests GC reports grants, personal fees as speakers' bureau member and advisory board member of Integra and Neuroptics, all outside the submitted work. FT received lecture fees from BD and ZOLL and personal fees as advisory board member of Nihon Khoden and Neuroptics, all outside the submitted work. M0 received grants from the Swiss National Science Foundation, and is a consultant and member of the Scientific Advisory Board of Neuroptics.

Patient consent for publication Not required.

Provenance and peer review Not commissioned; externally peer reviewed.

Supplemental material This content has been supplied by the author(s). It has not been vetted by BMJ Publishing Group Limited (BMJ) and may not have been peer-reviewed. Any opinions or recommendations discussed are solely those of the author(s) and are not endorsed by BMJ. BMJ disclaims all liability and responsibility arising from any reliance placed on the content. Where the content includes any translated material, BMJ does not warrant the accuracy and reliability of the translations (including but not limited to local regulations, clinical guidelines, terminology, drug names and drug dosages), and is not responsible for any error and/or omissions arising from translation and adaptation or otherwise.

Open access This is an open access article distributed in accordance with the Creative Commons Attribution Non Commercial (CC BY-NC 4.0) license, which permits others to distribute, remix, adapt, build upon this work non-commercially, and license their derivative works on different terms, provided the original work is properly cited, appropriate credit is given, any changes made indicated, and the use is non-commercial. See: http://creativecommons.org/licenses/by-nc/4.0/.

ORCID iD

Giuseppe Citerio http://orcid.org/0000-0002-5374-3161

\section{REFERENCES}

1 Sharshar T, Citerio G, Andrews PJD, et al. Neurological examination of critically ill patients: a pragmatic approach. Report of an ESICM expert panel. Intensive Care Med 2014;40:484-95.

2 Chen JW, Gombart ZJ, Rogers S, et al. Pupillary reactivity as an early indicator of increased intracranial pressure: the introduction of the neurological pupil index. Surg Neurol Int 2011;2:82.

3 Chen JW, Vakil-Gilani K, Williamson KL, et al. Infrared pupillometry, the neurological pupil index and unilateral pupillary dilation after traumatic brain injury: implications for treatment paradigms. Springerplus 2014;3:548.

4 Larson MD, Behrends M. Portable infrared pupillometry: a review. Anesth Analg 2015;120:1242-53.

5 Larson MD, Singh V. Portable infrared pupillometry in critical care. Crit Care 2016;20:161. 
6 Volpi PC, Robba C, Rota M, et al. Trajectories of early secondary insults correlate to outcomes of traumatic brain injury: results from a large, single centre, observational study. BMC Emerg Med 2018;18:52.

7 Han J, King NKK, Neilson SJ, et al. External validation of the CRASH and IMPACT prognostic models in severe traumatic brain injury. $J$ Neurotrauma 2014;31:1146-52.

8 Couret D, Boumaza D, Grisotto C, et al. Reliability of standard pupillometry practice in neurocritical care: an observational, doubleblinded study. Crit Care 2016;20:99.

9 Couret D, Simeone P, Freppel S, et al. The effect of ambient-light conditions on quantitative pupillometry: a history of rubber cup. Neurocrit Care 2019;30:492-3.

10 Olson DM, Stutzman S, Saju C, et al. Interrater reliability of pupillary assessments. Neurocrit Care 2016;24:251-7.

11 Hall CA, Chilcott RP. Eyeing up the future of the pupillary light reflex in Neurodiagnostics. Diagnostics 2018;8. doi:10.3390/ diagnostics8010019. [Epub ahead of print: 13 Mar 2018]

12 Larson MD, Sessler DI. Pupillometry to guide postoperative analgesia. Anesthesiology 2012;116:980-2.

13 Morelli P, Oddo M, Ben-Hamouda N. Role of automated pupillometry in critically ill patients. Minerva Anestesiol 2019;85:995-1002.

14 Shoyombo I, Aiyagari V, Stutzman SE, et al. Understanding the relationship between the neurologic pupil index and constriction velocity values. Sci Rep 2018;8:6992.
15 Jahns F-P, Miroz JP, Messerer M, et al. Quantitative pupillometry for the monitoring of intracranial hypertension in patients with severe traumatic brain injury. Crit Care 2019;23:155.

16 Aoun SG, Stutzman SE, Vo P-UN. Detection of delayed cerebral ischemia using objective pupillometry in patients with aneurysmal subarachnoid hemorrhage. J Neurosurg 2019;132:1-6.

17 Osman M, Stutzman SE, Atem F, et al. Correlation of objective pupillometry to midline shift in acute stroke patients. J Stroke Cerebrovasc Dis 2019;28:1902-10.

18 Ong C, Hutch M, Barra M, et al. Effects of osmotic therapy on pupil reactivity: quantification using pupillometry in critically ill neurologic patients. Neurocrit Care 2019;30:307-15.

19 Phillips SS, Mueller CM, Nogueira RG, et al. A systematic review assessing the current state of automated pupillometry in the NeurolCU. Neurocrit Care 2019;31:142-61.

20 Oddo M, Sandroni C, Citerio G, et al. Quantitative versus standard pupillary light reflex for early prognostication in comatose cardiac arrest patients: an international prospective multicenter doubleblinded study. Intensive Care Med 2018;44:2102-11.

21 Wilson L, von Steinbuechel N, Menon DK, et al. Outcome assessment after traumatic brain injury - Authors' reply. Lancet Neurol 2018;17:299-300.

22 Kompanje EJO, van Dijck JTJM, Chalos V, et al. Informed consent procedures for emergency interventional research in patients with traumatic brain injury and ischaemic stroke. Lancet Neurol 2020;19:1033-42. 